\title{
Écrits de détresse de femmes victimes de violences conjugales.
}

\author{
Samira Messaoudi ${ }^{1 *}$ \\ ${ }^{1}$ CREM Centre de recherche sur les médiations-Université de Lorraine : EA3476-34 cours Léopolod-CS \\ 35233-54052 Nancy cedex-France
}

\begin{abstract}
Résumé. Le présent travail porte sur les violences conjugales et leur mise en discours. Notre problématique vise à effectuer une analyse descriptive d'un certain type de situation communicative : la mise en discours de la violence conjugale à travers le témoignage numérique. Ce travail aura pour objectif de montrer et d'examiner comment la souffrance de femmes battues s'exprime sur la toile, et plus précisément au sein des forums. Quelles sont les spécificités des témoignages de femmes victimes de violences conjugales ? est-il possible, linguistiquement et sémiotiquement, d'établir des caractéristiques propres au témoignage sur forum lorsque la violence conjugale est racontée ? ainsi, nous observons des comportements spécifiques de mise en discours en faisant l'hypothèse qu'ils fassent l'objet de récurrences et, de ce fait, que leurs conditions de réalisation obéissent à certaines règles issues de leur appartenance au forum. Cette vérification de l'hypothèse tend, elle aussi, à mettre en avant la nécessité de l'établissement d'un corpus assez conséquent constitué de textes (dans notre cas les témoignages des femmes battues issus du Web). A cet égard, nous exploiterons notre objet d'étude à l'aide de deux logiciels, Cordial et Hyperbase.
\end{abstract}

\begin{abstract}
Distress writings of woman victims of domestic violence. The present work focuses on domestic violence and their speech. Our problematic aims to make a descriptive analysis of a certain type of communicative situation: the speech of conjugal violence through digital testimony. This work will aim to show and examine how the suffering of battered women is expressed on the web, and more specifically within the forums to answer the following questions: What are the specificities of testimonies of women victims of violence marital? Is it possible, linguistically and semiotically, to establish genderspecific characteristics of forum testimony when domestic violence is narrated? Thus, we observe specific behaviors of speech setting by making the hypothesis that they are the object of recurrences and, consequently, that their conditions of realization obey certain rules is-sues of their belonging to forums. This problem also tends to highlight the need for the establishment of a corpus of texts (in our case the testimonies of battered women from the Web). The qualitative and quantitative analysis of the content will allow us to work on very large digital corpora. In this respect, we will use our study object with two software programs, Cordial and Hyperbase.
\end{abstract}

\footnotetext{
${ }^{1}$ Samira Messaoudi : messaoudi.samira@outlook.com
} 


\section{Introduction}

La question de la violence conjugale jouit d'une complexité qui tend à la rendre difficile voire délicate à réduire ou cantonner à une seule acception, qui ne saurait englober la multitude de comportements, de caractères, d'actes, d'impacts, de signaux et de cycles. Bien qu'elle soit rattachée aux autres formes de violences qui sévissent dans la société, elle s'en distingue en raison de son caractère particulier. Le domicile, lieu de paix, de sécurité, de protection et d'intimé devient l'endroit du mépris, de l'agression, de la domination, du pouvoir paralysant et destructeur ainsi que de la violence de tous ordres et sous différents angles : physiques, sexuels ou sexués, psychologiques, économiques et verbaux.

L'avènement du numérique semble pouvoir faire tomber ces barricades. En effet, le partage des expériences est devenu nettement plus accessible autant par la possibilité de préserver l'anonymat en ayant recours à des pseudonymes, des noms d'emprunt afin d'éviter les jugements et l'identification, mais également du fait de la facilité à accéder aux différentes ressources. L'étendue de ces écrits d'écran est infinie : dès lors qu'un récit est édité et mis en ligne ou partagé sur des forums, il devient consultable par tous en tout temps et en tout lieu. Aussi, le sujet se trouve-t-il seul face à son écran pour raconter et se livrer : cette distance avec les potentiels récepteurs peut faciliter la mise en récit et donner libre cours aux personnes afin de parler de ce qui est qualifié en réalité d'inracontable.

Notre problématique vise à effectuer une analyse descriptive d'un certain type de situation communicative : la mise en discours de la violence conjugale dans le témoignage. Ainsi, notre objet d'étude est empirique : nous observons des comportements spécifiques de mise en discours en émettant l'hypothèse qu'ils fassent l'objet de récurrences et de ce fait, que leurs conditions de réalisation obéissent à certaines règles issues de leur appartenance au témoignage en ligne. Notre travail aura pour objetif de montrer et d'examiner comment la souffrance de femmes battues s'exprime sur la toile et plus précisément au sein du forum «briser la glace », quelles sont les spécificités langagières des témoignages de femmes victimes de violences conjugales ? Est-ce possible d'établir des caractéristiques linguistiques et discursives propres au témoignage de souffrance sur ce forum ? nous tenterons de définir quelles en sont les caractéristiques.

En premier lieu, il s'agira de porter un regard particulier sur les récurrences sémantiques de la violence en tenant compte des caractéristiques du forum «Briser la glace » et son impact sur le témoignage du public souffrant. Ensuite, nous tenterons de définir les caractéristiques du témoignage de souffrance (avec pour objet spécifique la violence conjugale) en procédant à différentes analyses syntaxiques, lexicales ou référentielles du corpus (étude des pronoms personnels, des temps verbaux, de la ponctuation, etc). Au vu de la taille du corpus récolté, une analyse assistée par ordinateur et à l'aide de logiciels se trouve être nécessaire voire indispensable. Les analyses qualitative et quantitative de contenu sont deux approches possibles permettant l'analyse des textes et discours. Nous exploitons notre objet d'étude à l'aide de deux logiciels : Cordial et Hyperbase. Cordial permet de décrire la partie morphologie de l'acte de témoigner dans un forum, tandis qu'Hyperbase est utile pour la partie thématique.

\section{Méthodologie de construction du corpus}

La récolte du corpus s'est effectuée sur un espace d'expression, d'écoute et de réconfort intitulé «Briser la glace », conçu pour les femmes battues, harcelées et humiliées. Il s'agit d'une mobi- 
lisation pour lutter contre les violences conjugales, générant la création d'un espace sécurisé et sécurisant répondant à un enjeu de société afin de faire reculer la honte, la suspicion et faire place à la libération de la parole et à la prise de conscience ; un dialogue interactif et ouvert à tous pour témoigner de cette violence trop souvent «silencieuse ». Nous avons puisé notre corpus au sein de la rubrique « témoignage » parmi les nombreux fils de discussions.

Chaque témoignage s'ouvrait sur un débat permettant aux autres de répondre dans un esprit de compassion et de partage afin d'apporter soutien et réconfort. Notre corpus est ainsi constitué de «700» témoignages de femmes victimes de violences conjugales. L'élaboration d'un corpus constitue l'une des premières étapes puisqu'elle détermine la validité du matériel recueilli et sa valeur de représentativité, ainsi que la fiabilité des opérations d'analyse. C'est ce que démontre Mayaffre (Mayaffre, 2005 : 10 ) en partageant l'idée que le corpus n'est pas l'outil de la recherche mais l'objet de la recherche, un objet ayant été construit et clôturé. Les étapes cruciales et primordiales lors de la récolte d'un corpus et pour une faisabilité de l'analyse sont : l'efficacité, le contrôle, l'accès et le partage, mais aussi la valeur de représentativité et la validité du matériel recueilli puisque le corpus est un objet ayant été construit et clôturé.

Pour pourvoir faire de l'analyse de discours, il convient de délimiter un espace discursif, autrement-dit, opérer une certaine clôture. En cela, se pose la question de l'élaboration et de la délimitation d'un corpus discussif. Les données authentiques traitant de la violence conjugale, qu'elles soient écrites ou orales, représentent une infinité de potentialité de perspectives d'étude. Ainsi, il est primordial de délimiter un champ d'exploration pour parvenir à obtenir un corpus assez conséquent pour qu'il soit le plus représentatif possible du phénomène que l'on cherche à analyser mais aussi et surtout de lui donner une certaine homogénéité.

\section{Outils de traitement du corpus}

Il existe diverses approches d'analyse du discours, chacune prenant en considération des aspects particuliers de l'objet discours. Le champ de l'analyse du discours est tellement vaste et morcelé que l'on pourrait même parler d'éclatement dans ce domaine. Par exemple, Benveniste s'intéresse aux phénomènes d'énonciation, Austin et Searle aux actes de langage, Ducrot aux connecteurs, à la présupposition et la polyphonie, Sperber et Wilson aux processus inférentiels, le Groupe Saint-Cloud au lexique, pour ne citer que ceux-là.

De plus, dans certains modèles, l'analyse du discours porte sur des énoncés isolés et/ou fabriqués pour les besoins de la démonstration. C'est le cas des analyses de Ducrot, de la théorie des actes de langage et de la théorie de la pertinence, entre autres. Pour les tenants de cette dernière théorie, le discours " n'est pas une catégorie pertinente » (Moeschler et Reboul, 1998 : 40), de sorte que l'analyse de la production langagière doit porter sur des énoncés, de manière indépendante. Dans d'autres modèles, comme le modèle modulaire de l'école de Genève, l'analyse porte sur le discours dans sa globalité. Étant donné ces difficultés, l'analyse du discours a un défi de taille à relever : celui de constituer son unité. Toutefois les problèmes de vues divergentes n'empêchent pas que l'analyse du discours soit possible en tant que technique permettant de questionner ce qu'on fait en parlant au-delà de ce qu'on dit.

Parmi les approches du discours les plus en vue ces 50 dernières années, on peut retenir l'analyse textuelle du discours, l'analyse de contenu du discours, l'analyse énonciative du discours, l'analyse modulaire du discours, ou encore l'analyse pragmatique du discours. Certaines approches en analyse du discours vont insister sur l'analyse statistique textuelle; on parlera aussi de logométrie (logos = discours ; métrie = mesure). Le discours est conçu comme un ensemble de données textuelles auxquelles l'analyste apportera la plus grande attention (recherche d'attestation, fréquence d'utilisation des mots, distribution du vocabulaire, calcul des co- 
occurrences, etc). Cette approche utilise des logiciels scientifiques : Hyperbase et Cordial. Les différents extraits récoltés ont été lemmatisés avec un logiciel de traitement de texte pour une meilleure exploitation, puisque le nombre de témoignages était assez conséquent. Les analyses qualitative et quantitative de contenu sont deux approches possibles permettant du discours sur forum, tandis qu'Hyperbase est utilisé pour la partie thématique. Le logiciel «Cordial » nous a permis de réaliser ces opérations qui ont transformées le texte brut dans sa matière première tel qu'il a été récolté en un texte lemmatisé pour permettre l'analyse. Ce logiciel permet de faire bien d'autres exploitations en plus de ses fonctions premières : établir une analyse statistique de toutes les caractéristiques stylistiques des textes (les totaux, les moyennes, les phrases, la ponctuation, la morphologie, l'usage, les noms, les pronoms, les verbes, les adjectifs, les adverbes, les types et le style). Il informe quant au traitement des expressions, à la fonction dans la phrase, à la place hiérarchique du mot dans l'arbre syntaxique et à la classe sémantique à laquelle le mot se rattache. La délimitation d'exploitation des données sur support numérique suffisait à délimiter notre champ d'étude, mais il nous a paru pertinent de nous intéresser à un champ réduit parmi, les divers supports numériques qui s'offraient à nous (blogs, chats, réseaux sociaux, plateformes numériques, etc), à un support unique : celui du forum.

Le forum est un espace virtuel destiné à l'échange de messages sur différents thèmes, mais il est aussi et surtout un document dynamique produit collectivement de manière interactive sous la forme d'une correspondance électronique archivée automatiquement, comme le définit (Marcoccia, 2001 : 15). De ce point de vue, nous disposons d'un corpus idéal pour l'analyse des conversations et l'analyse du discours, car il s'agit tout d'abord d'échanges authentiques produits en l'absence de l'analyste qui les enregistre, et ensuite, d'une homogénéité définie par leur mise en mémoire et par le dispositif ou l'institution qui a assuré cette mise en mémoire, à la lumière des travaux de (Maingueneau, 1991)

\section{Caractéristiques émergentes des témoignages: L'éminence narrative dans les témoignages des femmes battues}

Les témoignages ont une dominance narrative et suivent un certain schéma. Ils racontent des événements et rapportent des paroles. De ce fait, comprendre un témoignage, c'est analyser l'histoire mais surtout analyser la narration : c'est la façon de raconter qui donne réellement ses significations à ce type de discours. Selon les recommandations du forum, les témoignages au sein de «briser la glace » permettent la prise de parole, de contact mais aussi l'extériorisation. De plus, ils prennent en compte les expériences pour contrer la violence, raconter, narrer, convaincre et témoigner. Le témoignage constitue un discours alternatif exprimant une vérité concrète et des émotions objectivées, en respectant une distanciation du discours de haine.

Comme l'avancent à juste titre Claudine (Moïse et Claire Hugonnier, 2019 : 7), en référence aux propos de J.-M. Adam, le témoignage sous la forme d'une narration use de caractéristiques du récit oral dans une intention de vérité et à partir de deux procédés essentiels, une construction narrative autour de faits précis et crédibles et des éléments émotionnels objectivés. Les témoignages de femmes battues se caractérisent par des récurrences narratives et une logique causative qui montre l'enchaînement des évènements, la répétition, la généralisation. Ces derniers ancrent les récits de vie dans une expérience de partage. Afin de préciser notre démarche, nous nous attardons sur les différentes séquences ainsi que sur la construction de la narrativité.

Dans le sillage des classements de textes, nous nous référons J.-M. Adam. Comme il le nomme «type de discours », ou " forme de discours », est une catégorie qui classe les textes en se fondant sur des critères linguistiques et observables à l'intérieur d'un même texte. En effet, 
un texte comporte et renferme soit des séquences argumentatives, descriptives, dialogué ou narratives, soit et en vue de son caractère ouvert, il peut faire preuve de différentes séquences textuelles de types différents comme le démontrer ces exemples tirés du corpus :

«Je tiens à raconter mon histoire, pour pouvoir aider, et pour montrer le bonheur que je vis actuellement en ayant eu le courage de partir »

«Mon histoire a duré 9 ans... ça a commencé par un coup »

«Cette histoire réelle est la mienne. Et parce que les mots sont parfois un médicament, j'ai rédigé un livre-témoignage. Mais à ce jour, aucun des dix éditeurs que j'ai contactés ne semble intéressé par mon histoire. J'ai le sentiment d'être transparente »

«Voici mon histoire que je résume...»

«Je souhaitais raconter mon histoire pour à la fois peut-être me libérer et pour aider des femmes qui me liront. »

«Cette histoire a commencé comme toutes les autres ou presque, en tous cas ça a été fusionnel dès les premiers instants. »

«Pour résumer mon histoire, ce soir du 16.09.2002 ma vie a basculé lorsque je me suis refusée à mon conjoint qui était très alcoolisé. »

« Je tiens à raconter mon histoire, pour pouvoir aider»

«J'ai les larmes qui coulent, je me reconnais dans certaines histoires alors je vais vous raconter la mienne.»

En effet, les femmes battues racontent une histoire, par conséquent les séquences narratives sont à forte prédominance dans la structure de nos témoignages. C'est d'ailleurs cette suprématie et prévalence qui fait d'eux des témoignages. L'histoire de ces femmes victimes de violences conjugales est toujours expressément et clairement annoncée dans leurs écrits à l'aide du verbe « raconter ».

\subsection{La séquentialité}

Le texte narratif se conforme à un certain schéma «type » que nous avons évoqué brièvement auparavant et dont J.-M. Adam est à l'origine. Il est à préciser que ce schéma dont il est question n'est pas identique dans tous les textes et que son ordre naturel tolère des modifications ou des suppressions qui n'impactent pas pour autant la dominance narrative. Il existe cinq moments de tout procès à l'intérieur d'une séquence narrative ou d'un texte narratif tout entier : la situation initiale, le nœud, la réaction ou évaluation, le dénouement et la situation finale. Nous avons analysé ces témoignages selon les caractéristiques citées ci-dessus. Au sein des histoires aussi variées soient-elles, la situation initiale met en jeu le début de l'histoire d'amour des personnes ainsi que la quiétude et la sérénité qui régnait dans leurs couples fusionnels ; une sorte de représentation et d'idéalisation de la perle rare qu'elles ont rencontré :

«Il dirigeait tout avec brio avant de devenir une entreprise de destruction d'âme. J'étais folle amoureuse de lui.»

«On veut retrouver et garder la vie d'avant ...»

«Il est l'homme dont j'étais éperdument amoureuse. Prince charmant au début, extrêmement serviable et gentil avec son entourage, cultivé et ayant fait des études, pour moi il ne pouvait pas être violent, ça devait être de ma faute...»

«Il était une fois une belle histoire d'amour.»

«Je me détache de ma famille pour me tourner corps et âme vers ce premier amour. » 
«Mon entourage se félicitait de cette rencontre qui me stabilisait comme on dit, et se pâmait d'admiration pour cet homme plein de sollicitude, qui m'apportait sécurité, réconfort, statut social et vie trépidante. Eperdue de reconnaissance »

« Je l'ai rencontré et aimé pour être aimée... gentil, attentionné, sensible à ma souffrance sur ma vie auprès de ma famille... »

À travers ces quelques extraits du corpus, nous constatons que la plupart des femmes commencent leurs histoires par des débuts prometteurs d'un couple idéal, fusionnel, avec l'évocation de la passion, du bonheur, de la quiétude, de l'écoute, de la sérénité et de la sécurité dans qu'elles ressentaient, ensuite elles basculent dans une spirale infernale, dès l'apparition des premiers coups, du malheur et la torture qu'elles subissent. A ce stade précis, elles mettent en avant soit l'envie de s'en sortir et d'oublier les années de souffrances, soit au contraire l'envie de rester et de changer l'inchangeable : « je lui ai donné, non pas une seconde, mais une énième chance par amour ». Le conjoint qui se représentait tel un «l'étalon, prince charmant, homme dévoué et âme sœur» se transforme en un monstre, fou, violent et alcoolique. Ainsi, avant l'élément déclencheur, les récits sont imprégnés et inondés d'éléments d'exaltation et de satisfaction sous forme de forte utilisation d'adjectifs et de noms tels que : "bonheur », "plaisir », «bien-être », «heureuse », «contente ». Survient ensuite, l'annonce de l'explosion de la violence et la phase d'accumulation où subsistent un grand nombre d'adjectifs descriptifs et dévalorisants envers elles-mêmes : " soumise, faible, dominée, passive, dépendante et victime ». Le changement de situation intervient également à travers la locution adverbiale «alors que » qui marque l'opposition la locution « tout cela » qui fonctionne comme anaphore présomptive, pour passer à quelque chose de nouveau et le verbe transformer qui évoque aussi le changement des états :

«C'est alors que je l'ai vu comme une larve immonde. Moi si faible, si fragile, si naïve, si craintive... »

« .. Il apprenait à connaître mes faiblesses ... Je me croyais forte. »

«C'était mon patron et mon mari, ma vie a été vécue sous l'abus de pouvoir, l'abus de faiblesse et la maltraitance. »

«Tout cela se passait la plupart du temps à huis clos: dénigrement, coups, menaces, dépendance financière, intimidation. Les témoins c'était lui qui les choisissait pour leur faire constater ma bêtise, mon immaturité, ma faiblesse...»

«C'est la première fois que je me sens faible, telle une merde. »

«Moi qui étais une femme indépendante, courageuse, débrouillarde, je me transforme jour après jour en une femme soumise, peureuse, sans beaucoup de volonté. "

« Je vivais cloitrée, terrorisée, soumise par la force et la manipulation, désespérée, et incapable de trouver un moyen de lui échapper. C'est en regardant le film " L'emprise" que je me suis rendue compte que j'avais été victime de violences conjugales. »

Enfin, la phase de dénonciation, la femme battue demande de l'aide et tend à se désencombrer du poids du silence dans lequel elle s'était murée. Elle essaye tant bien que mal de mettre fin à ce calvaire en demandant de l'aide et se définit comme « heureuse, libérée et délivrée » :

« Aujourd'hui je suis libre !!!! Je vais où je veux, quand je veux avec qui je veux ! Je travaille et j'ai mon permis! Je me retrouve avec mes amis ! Je profite de ma vie et de ma jeunesse !! JE SUIS HEUREUSE ! J'ai choisi d'être aidée par un coach pour travailler l'estime et la confiance en moi car j'ai été détruite et abîmée par cette relation. »

\subsection{Les temps verbaux}

Les témoignages de femmes battues se répartissent entre récits et discours selon la présence ou l'absence de temps verbaux et des embrayeurs. En ce qui concerne le discours, les temps des 
verbes s'organisent par rapport au présent : passé composé, imparfait --->présent---> futur simple, futur antérieur. En ce qui concerne le récit, les temps verbaux s'organisent par rapport au passé simple : passé antérieur, plus-que-parfait, imparfait--->passé simple--->futur du passé (identique au conditionnel). Les temps verbaux demeurent des éléments fort intéressants et très révélateurs en analyse de discours, de ce fait ils méritent une attention particulière. Nous avons vérifié quels sont, dans notre corpus, les temps verbaux qui sont les plus utilisés et ceux qui le sont moins :

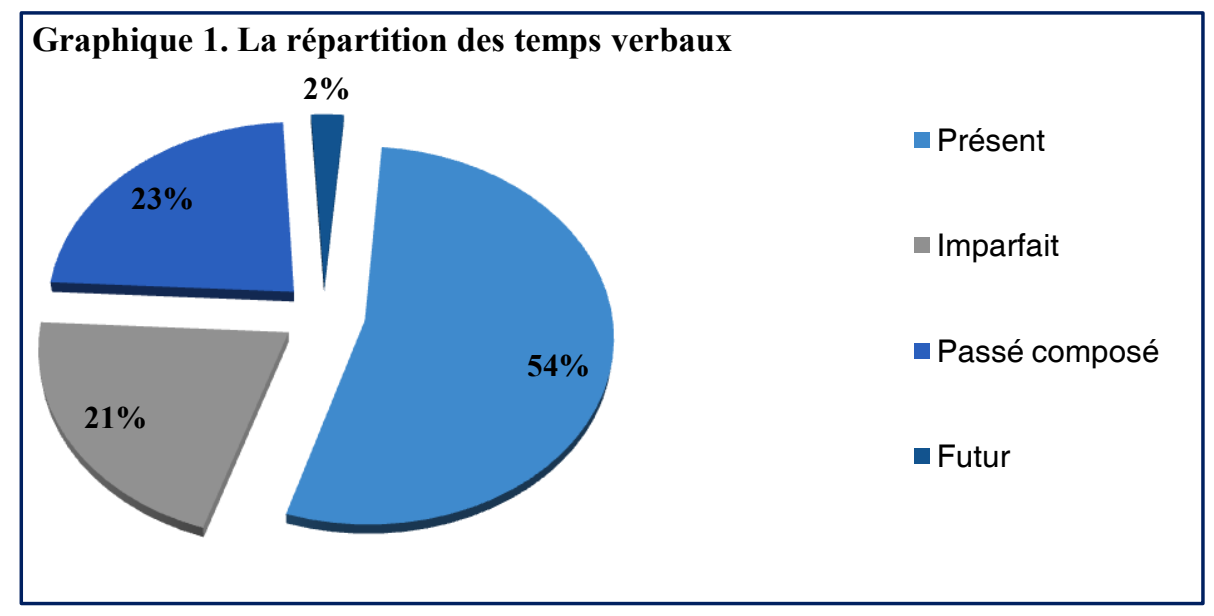

Fig.1. La répartition des temps verbaux dans le corpus des femmes battues.

Ce circulaire permet de constater que les temps dominants au sein des témoignages des femmes battues sont le présent, le passé composé et l'imparfait. Trois temps qui permettent de raconter une histoire, celle de leurs tragiques mésaventures durant lesquelles la présence de ces trois temps de narration paraît évidente dès lors que nous avions établi la dominance narrative qui prédomine. Le présent domine à valeur de 54,00\% et c'est pour cela que nous nous sommes attardés sur son utilisation au sein du corpus. Il prédomine dans le corpus et qui endosse deux valeurs en alternant entre présent de l'énonciation et présent de la narration. D'abord, le présent d'énonciation ancre le témoignage dans la situation d'énonciation en marquant une pause dans la narration. C'est le moment où le narrateur « femme battue » oublie un temps l'histoire racontée et témoigne tout en introduisant dans le cours de son récit une réflexion qui lui vient à l'esprit. Au sein de ce corpus, le présent de l'énonciation évoque le moment où les femmes s'expriment sur leurs états, pensées, douleurs et souffrances en mettant de côté la narration des faits rapportés dans le récit. Le présent de l'énonciation, comme le temps du témoignage dans le corpus est ancré dans la situation d'énonciation et représente le présent du moment où l'on parle.

« Les maux sont inscrits dans les mots, marquage au fer rouge... »

« Je veux, par mon témoignage, apporter de l'espoir à toutes celles qui vivent l'enfer »

«J'ai les larmes qui coulent, je me reconnais dans certaines histoires alors je vais vous raconter la mienne»

« Je suis une jeune de 24 ans »

« Je tiens à raconter mon histoire, pour pouvoir aider .»

« Je suis bouleversée...» 
« Je veux témoigner surtout principalement du caractère caché de l'enfer, du mal et de la malveillance fondamentale qui nourrissait mon ex-mari. »

« Aujourd'hui si je décide de témoigner »

«Aujourd'hui certes je vis"chichement»

«je suis enfin HEUREUSE, libérée, délivrée de la peur »

Ensuite, le présent de narration qui rapporte au présent des temps passés. Il met en relief des événements d'un récit passé afin de donner une impression d'actualité, un effet de direct et des actions plus vivantes. Une impression qui défait la distance temporelle entre le moment de l'histoire qui est racontée et la narration, pour permettre une meilleure visualisation de ce qui se dit. C'est un présent qui intervient dans un contexte de temps au passé, l'action se situe dans le passé et se raconte à l'aide de verbes au présent. Les récits au passé sont coupés de la situation d'énonciation et par conséquent le présent de narration permet de visualiser au mieux ce qui est dit comme si cela était en train de se passer au moment où le co-énonciateur lit ou l'énonciateur écrit :

«Et je reste seule sans en parler. Puis j'essaye petit à petit d'en parler... Mes amies me conseillent de le quitter et d'aller porter plainte mais je ne les écoute pas. Je pense aux moments beaux qu'il me fait vivre parfois... Et puis je l'aime. Et puis j'ai peur aussi...»

Alors je décide de rester, d'ailleurs je suis encore avec aujourd'hui. Et je deviens moi-même violente. » « Je souffre et je me sens coupable»

« Je vais mieux, je suis fière de moi, de ce que je suis devenue, j'ai appris à m'affirmer, à vivre. Je suis indépendante et je tiens à le rester et je pleure, pleure de tristesse, pleure de peur, pleure et pleure encore. »

La présence des deux temps du présent, présent narration et présent de l'énonciation, représente deux différentes temporalités construites qui accordent des statuts aux femmes battues énonciatrices. Tantôt elles permettent de créer un effet de vif et d'actualité, tantôt elles respectent le moment de la narration et de la succession des faits. Une souplesse qui permet aux femmes de narrer et d'énoncer en combinant les deux simultanément ou successivement.

Le passé composé permet d'exprimer un fait passé entièrement et achevé. Un aspect accompli qui s'oppose souvent à l'aspect inaccompli de l'imparfait auquel il est souvent associé. En effet, il peut être d'une part, strictement passé et de l'autre lié au présent. Sa valeur est l'actualité, les traits accompli, cursif, itératif, et limitatif ou bien non accompli décrivant une action située dans une période passée dont les conséquences peuvent être appréciées et mesurées au présent. Il est souvent employé dans des énoncés ancrés dans la situation d'énonciation. Il prend parfois la valeur d'un présent accompli mais peut peut également exprimer une action antérieure par rapport au présent, traduire un futur proche, présenter un fait comme une vérité générale ou remplacer le passé simple. Le passé composé a une valeur d'accompli et c'est cette particularité qui caractérise son incapacité narrative comme l'avance à juste titre Oswald Ducrot : «L'aspect est [...] accompli si le procès est antérieur à la période dont on parle, mais si on veut signaler sa trace dans cette période » (Ducrot, 1995, 682-696). L'aspect est accompli lorsque le procès se présente comme achevé au moment qui sert de repère temporel et c'est à ce momentlà qu'on envisage alors le résultat du procès. Au sein du corpus, nous avons noté quelques particularités du passé composé. Il marque la prise de décision lors de la séparation, il est suivi d'un marqueur temporel "nombre d'années », il permet aussi de constater et de décrire des faits récents :

«J'ai fini par partir, après $\mathbf{1 0}$ ans d'humiliations, de violence psychologique, d'isolement puis de harcèlement, prix de mon départ... » 
« $\mathrm{J}$ ai vécu 13 ans dans la violence conjugale » «enfin j’ai réussi à m’en sortir parce que $\mathbf{j}$ ai brisé le silence. »

«J'ai quitté le domicile conjugal il y a 6 mois après avoir vécu pendant 10 ans des violences physiques et psychologiques. »

« J'ai vécu 4 ans de ma vie avec un homme violent. »

« Mon compagnon m'a mise à la rue après plus de 20 ans de vie commune.»

Nous avons pu remarquer que le futur est quasi inexistant, laissé à l'abandon et ne représente que $2 \%$ par rapport aux autres temps verbaux. Cette absence s'explique par le dénouement de l'histoire, qui démontre que les femmes battues n'arrivent pas à se projeter dans l'avenir ou à l'envisager sereinement. Des moments stériles, douloureux, injustifiés et insupportables qu'elles ne sont plus capables d'endurer davantage. Un cheminement angoissant et un univers si chaotique que les femmes plongent dans l'obscurité et ne savent plus comment s'en sortir puisqu'elles s'abandonnent à ne voir que le malheur aussi minime soit-il. Le sens subsiste dans les absences autant que dans les présences, de ce fait nous avons relevé toutes les occurrences des verbes conjugués au futur afin d'observer leur contexte d'apparition. Un temps utilisé à une aussi petite échelle mais tout de même exprimé, alors dans quelle situation l'est-il ?

« J'ai peur qu'un jour ça aille trop loin et qu'il finisse par mettre à exécution ses menaces... Que pourrai-je faire ?»

« Je ne pourrai pas le prouver. Je ferai une main courante mais est ce que ça suffira à me protéger?»

« Je ne pourrai plus jamais me regarder dans une glace sans voir cette honte... ».

Nous constatons à l'aide de ces exemples que l'utilisation du temps du futur dans le corpus renvoie aux énoncés interrogatifs ainsi qu'à la négation, de ce fait nous émettons comme hypothèse que le futur de ces femmes battues demeure inenvisageable puisqu'elles se cantonnent à déplorer leur sort, et évitent de s'attarder sur une éventuelle nouvelle vie sans violences.

\section{Les traces de l'énonciation}

L'énonciation prend en considération la position de l'énonciateur et du locuteur dans sa production d'un énoncé précis, aussi la langue n'est plus qu'un simple objet interne, elle jouit d'un dynamisme, d'une stratégie, d'un agencement conscient et réfléchi. L'énonciation est définie comme « la mise en fonctionnement de la langue par un acte individuel d'utilisation » (Benveniste, 1970 : 12-18 ). Il précise que l'étude de l'énonciation d'un point de vue linguistique tend à relier les formes linguistiques à l'acte d'énonciation, et vu que l'énonciateur en fait partie nous nous intéressons de plus près à sa position quand il produit un énoncé.

En effet, un pronom personnel désigne celui qui parle, ou celui à qui le locuteur s'adresse, de plus du point de vue du lecteur les pronoms sont des indices de l'énonciation. Identifier la personne à laquelle ils réfèrent permet de savoir qui parle à qui, par conséquent nous analysons les pronoms personnels qui nous permettront de constater comment le locuteur se positionne-t-il comme sujet dans l'énonciation au sein de ce corpus et quelle place accorde-t-il à ceux à qui il s'adresse ? il existe plusieurs notions à travers lesquelles un locuteur se définit comme étant un sujet dans le champ de l'analyse du discours. Des unités qui ne prennent de sens que lorsqu'elles sont rattachées à un acte particulier d'énonciation nommées : les embrayeurs.

Les pronoms personnels sont des aspects indiciels du langage, de ce fait nous nous y intéressons dans notre corpus de femmes battues. Un soin particulier a été accordé au pronom le plus utilisé dans le corpus «je ». Le graphique ci-dessous fait apparaître clairement le grand nombre 
d'utilisations du pronom personnel « je ». L'emploi de ce dernier désigne dans notre corpus la personne qui l'emploie en racontant sa propre histoire.

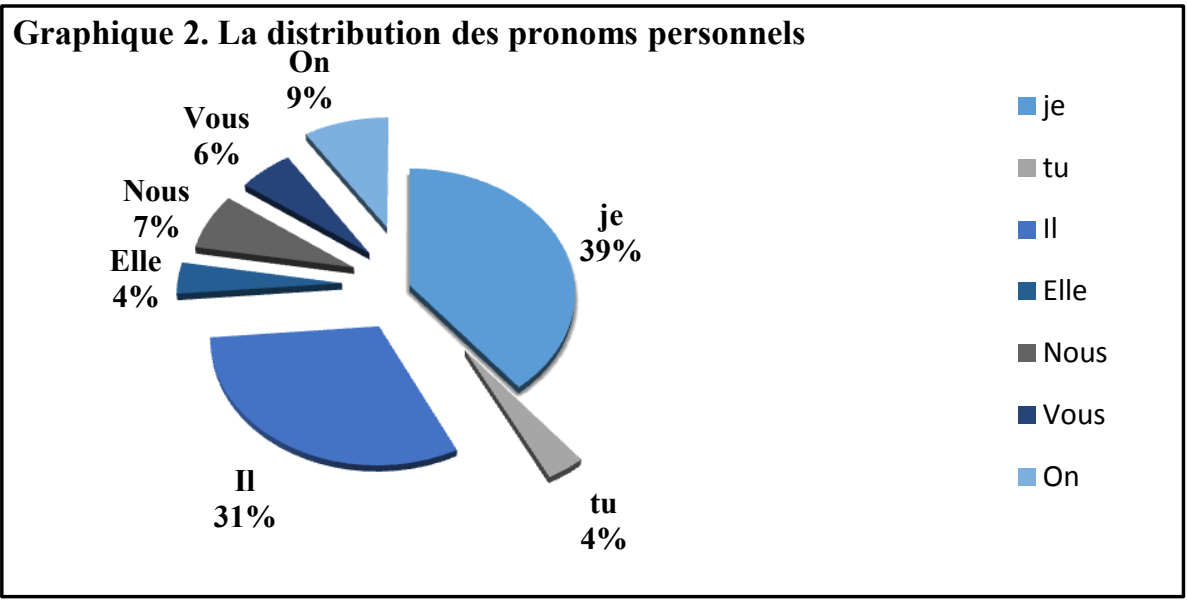

Fig. 2. La distribution des pronoms personnels dans le corpus des femmes battues.

Nous avons extrait un nombre d'exemples du «je» témoignant de l'énonciation en cours dans le corpus :

« moi, ça fait trop longtemps que je me tais.je n'en peux plus... je suis au bout de mes forces, et je sens que rien n'est fini...je sors la tête pour respirer en sachant que j'ai pas fini de boire la tasse... »

« Je ne pouvais pas baisser les bras j'avais 3 petits bouts qu'il fallait éduquer, nourrir, habiller suivre la scolarité etc. »

« Je ne savais pas où me confier. C'est très difficile d'en parler. »

«Je n'ai plus d'imagination pour me projeter ailleurs. »

« Je ne crois pas qu'il va changer et sincèrement je ne veux même pas qu'il change pour être mieux avec moi »

Une constatation qui émerge après analyse et qui se répète : celle de l'emploi du « je » dans des phrases négatives, des phrases qui représentent un très grand taux selon les statistiques du logiciel Cordial qui en relève un très grand pourcentage, ainsi que selon les occurrences d'Hyperbase puisqu'une occurrence représente le nombre de fois où le pronom personnel apparaît dans le corpus en mesurant son indice de densité._Le «je» est majoritaire dans l'ensemble du corpus, en relation directe avec le type de discours qu'est le témoignage personnel puisque c'est le « je » de la femme qui témoigne. Le « il» vise à relater les faits opérés par l'agresseur. Ensuite, «nous » est le plus souvent associé au passé "normal », avant que les violences ne commencent. Les pronoms personnels expriment différentes valeurs et différents référents : «tu » du DR, le «tu » générique, le « il » impersonnel», le « nous » inclusif et exclusif. Il existe deux plans de l'énonciation : l'un de l'histoire et l'autre du discours. Dans notre corpus des femmes battues qui penche vers le récit, émerge une basse fréquence de l'utilisation du pronom « tu $»$.

La dualité « je / tu » « je » au sein d'une énonciation est très significative, comme l'avance à juste titre Benveniste, le pronom personnel «tu » n'est employé qu'à très basse échelle comparé 
aux autres puisqu'il ne représente qu'un taux de $4 \%$ parmi l'ensemble des pronoms personnels. Ce taux ne signifie pas qu'il a moins d'importance, qu'il est très peu utilisé mais qu'il est sousutilisé par rapport aux autres pronoms. Une question subsiste, pourquoi ?

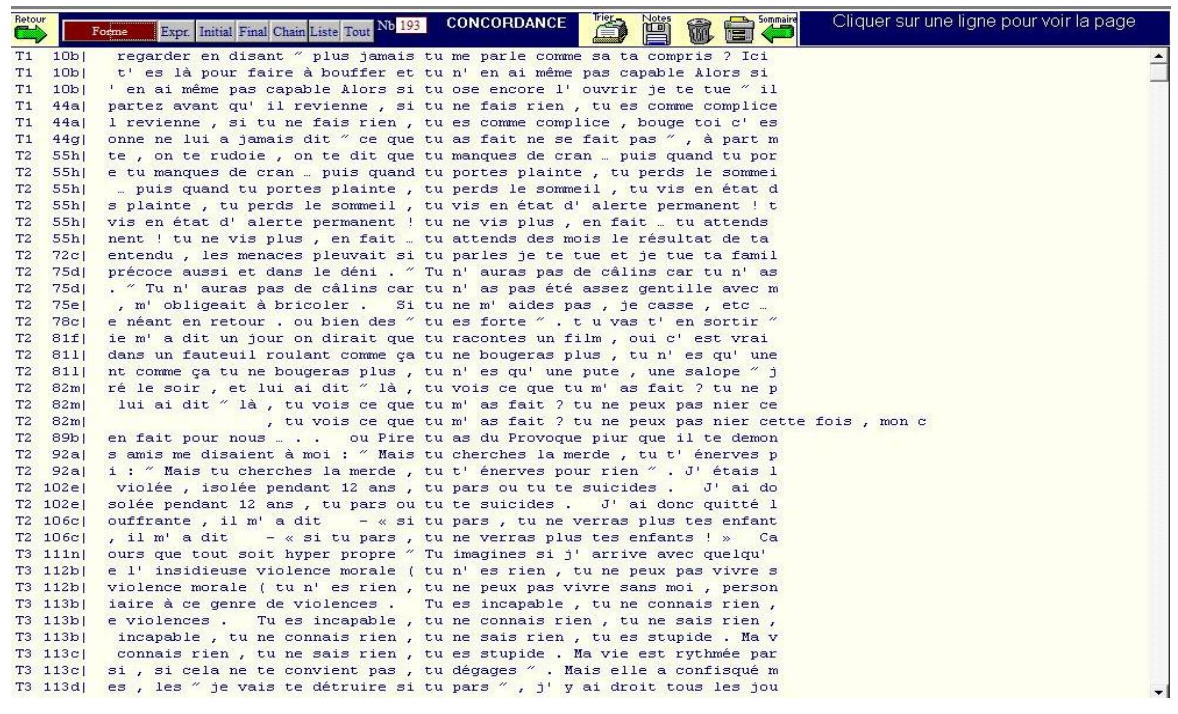

Annexe 1. Tableau des concordances du pronom personnel « tu » dans le corpus des femmes battues.

D'après le tableau des concordances et à l'aide des exemples tirés du corpus, nous constatons que le pronom personnel «tu » renvoie à plusieurs personnes, puisqu'il représente trois valeurs différentes comme le démontre ces exemples :

«Qu'est-ce que tu racontais à ta copine durant tout ce temps au téléphone? ».

« elle me racontait le mariage de sa cousine»

«il a rappelé le dernier numéro afin de s'assurer qu'il s'agissait bien d'elle et que je ne lui mentais pas. »

« il m'a dit "tu te rends compte si tu m'avais menti ce qu'il te serait arrivé ? Mais ce n'est pas pour autant que je vais te le pardonner. Vous parliez de moi? Tu te plaignais? hein! »

Le « tu » qui figure dans le premier discours rapporté a pour but de raconter l'histoire de la personne ayant vécu la situation, les dialogues permettent une immersion dans le passé afin de mieux visualiser les scènes qui se sont déjà déroulées et que les femmes réinvestissent au profit du témoignage. Des souvenirs douloureux qu'elle a vécus à un moment donné et qu'elle essaye de nous raconter. La femme battue s'adresse à l'homme qui lui a porté des coups et qui est violent envers elle en écrivant le «tu », elle n'attend aucune réponse de sa part, elle est consciente qu'il ne lui répondra pas, ce qui donne une sorte de dialogue à sens unique où la femme fait comme si l'homme lisait ces posts et témoignages. Ainsi l'utilisation du «tu » exclut le lecteur des faits qui se déroulent puisque la femme s'adresse à l'homme qui l'a battue et non pas à la communauté, elle exclut tous les autres.

«Ces moments où tu revois ces scènes destructrices en boucle »

« Tu pleures. Tu pleures comme si tu les revivais à nouveau. Tu te sens toujours humiliée et rabaissée. » «Des souvenirs qui resteront à jamais gravés en mémoire et qui ne pourront s'effacer ni avec le temps ni avec l'oubli. » 
Dans ces cas, le «tu » ici générique vient remplacer le « je » afin de distancier les propos écrits de manière à ne pas trop en souffrir, une façon de s'en détacher et une tentative de ne plus revivre le moment. Cependant certaines femmes battues ou qui l'étaient ou le sont encore dans certains cas ont réussi à prendre du recul et témoignent sur ce forum en utilisant le « je » à la place du «tu ». Est-ce déjà une victoire ? Est-ce une autre étape qui s'annonce ? Est-ce le début d'une nouvelle vie?

« Alors voilà, tu as réussi à me faire arrêter le travail, à rester à la maison, tu m'a isolée de ma famille et de mes amis mais j'ai quand même repris le dessus. Toi tu as touché le fond.»

«Moi, je m'en suis sortie, j'ai eu la garde des enfants, j'ai réussi à faire ma vie tandis que toi, tu sombres dans ton alcool et que personne ne veut plus t'approcher. »

«Tu m'as détruite, tu m'a rabaissée, tu m'a humiliée, tu me domine et me commande »

«C'est à ce moment que tu t'es rendu compte que je n'avais plus peur de toi, tu avais sûrement dû voir dans mes yeux que tu ne me donnais plus qu'envie de vomir »

Un cas très rarement utilisé mais qui apparaît dans certains témoignages : des femmes battues qui utilisent le «tu » non pas pour désigner les femmes à qui elles s'adressent ou les membres de la communauté de ce forum qui leur est dédié, mais pour désigner leur mari violent, conjoint, compagnon ou petit ami, en d'autres termes le porteur de coups. Elles s'adressent ouvertement dans leurs témoignages à cette personne comme si elles lui écrivaient une lettre ou comme si elles avaient envie de lui adresser un message ou de se faire lire par leur agresseur. Étant donné qu'il s'agit de témoignages, nous avons noté l'utilisation du « vous » provenant du support numérique qui permet à cette communauté de femmes battues de s'exprimer ; elles s'adressent les unes aux autres et à tous ceux et à toutes celles qui lisent leurs publications. Une observation fondée parce que nous nous trouvons dans le cas d'un corpus de témoignages et ce genre a pour but de « raconter une histoire », ce qui explique la présence du pronom personnel « vous ».

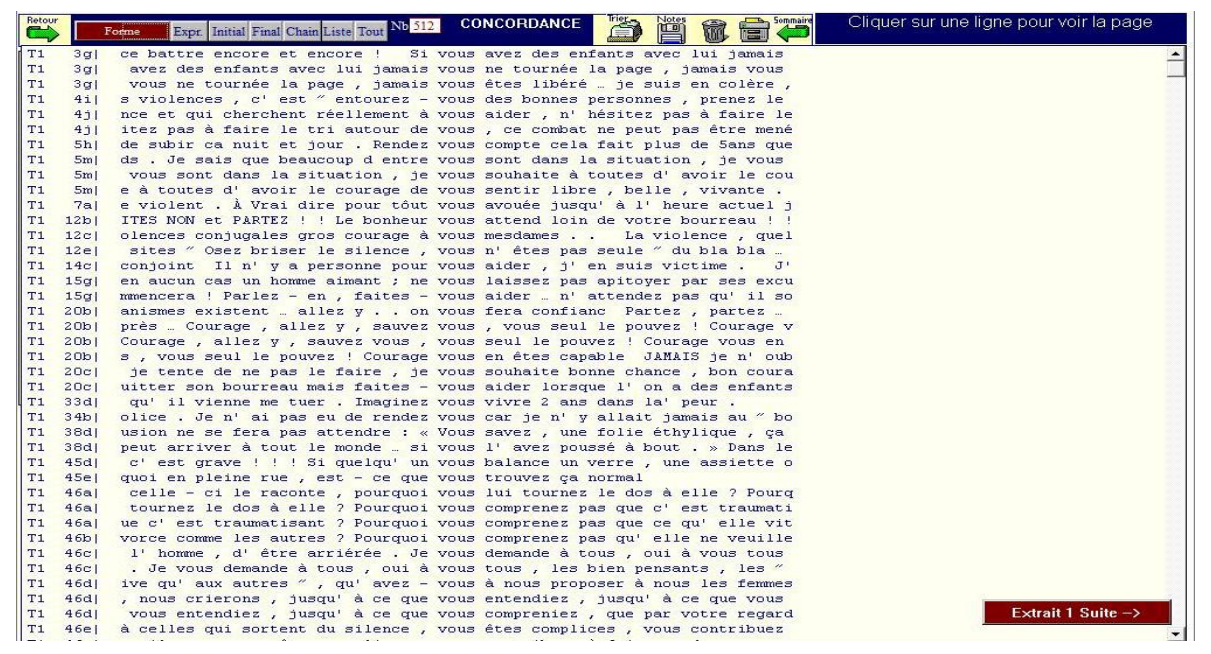

Annexe 2. Tableau des concordances du pronom personnel « vous » dans le corpus des femmes batues.

La visualisation des concordances dans l'annexe ci-dessus montre plusieurs emplois du pronom personnel « vous » comme le montre ces exemples :

« Je vous comprends et je compatis avec vous, j'ai été victime du même homme » Ainsi le «vous » renvoie aux femmes qui sont elles aussi battues. 
«Le bonheur vous attend loin de votre bourreau !!! J'espère vraiment que mon témoignage aidera des femmes victimes de violences conjugales gros courage à vous mesdames ... »

Le « vous » dans les exemples ci-dessus est adressé aux autres femmes battues en particulier. Les exemples suivants, sont au sein d'un discours rapporté et totalement détaché de la situation d'énonciation :

« Je suis désolé vous devez regagner votre domicile conjugal, on ne peut intervenir tant qu'il ne vous a pas fait quelque chose de grave. »

«Nous sommes navrés de vous dire qu'il ne peut y avoir de poursuites au vu du manque de preuves que vous nous avez ramenées. »

«Madame, nous ne pouvons pas inculper votre homme parce que vous l'avez décidé.»

Dans ce cas le « je » et le «nous » réfèrent à la justice, à des policiers et au corps judiciaire et «vous » réfère à la femme battue qui a été porter plainte. Le pronom « vous » est souvent adressé aux familles des femmes battues, entourage, parents, amis et collègues de travail :

«Cela fait des années que je vous mens en le faisant passer pour l'homme parfait. »

« J'aimerais vous faire plaisir, le laisser et quitter le domicile mais je n'y arrive pas. »

« Je me battrai afin de vous donner un avenir meilleur, je n'accepte plus que vous preniez des gifles et des coups de poing alors que vous n'avez rien fait. »

\subsection{La subjectivité dans la ponctuation}

Le corpus présente un nombre très conséquent d'éléments subjectifs dont les signes de ponctuation. Ils ne servent pas seulement à structurer un texte mais aussi à en démontrer la subjectivité lorsqu'il s'agit de discours, de pensées, de sentiments ou d'émotions.

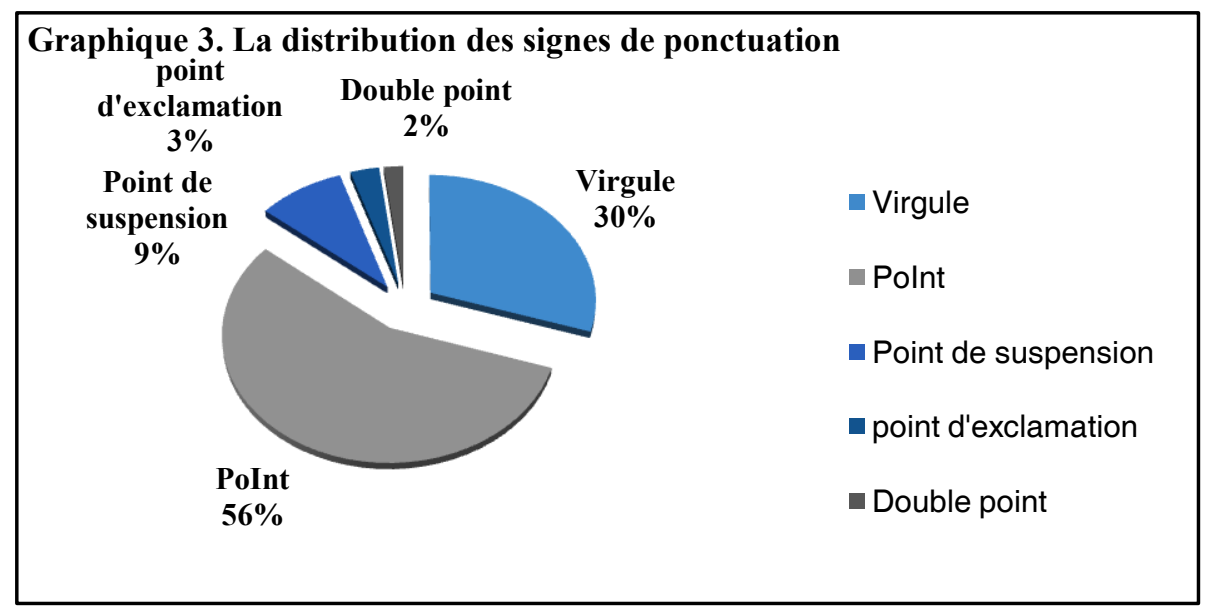

Fig.3. La distribution des signes de ponctuation dans le corpus des femmes battues.

Les points de ponctuation dominants, comme le montre le graphique, sont premièrement «le point » à valeur de 56\% par rapport à l'ensemble de ponctuation. Deuxièmement, « la virgule » à valeur de $30 \%$ par rapport à l'ensemble de ponctuation. Les femmes battues témoignent en utilisant des phrases simples et courtes et ne ressentent pas le besoin de faire beaucoup de coupures ou de reformulations :

«Il avait déjà beaucoup d'emprise sur moi, mais je ne m'en étais pas encore aperçue. » 
« l'emprise s'est fait sentir de plus en plus, je n'avais pas le droit d'être belle ou bien habillée,( j'ai même fini par croire que j'étais moche) ni dedans ni dehors, uniquement en famille, il m'a isolée de toute ma famille ou presque, de beaucoup d'amis. »

«Le permis en poche, un bout de liberté se profilait à l'horizon.

« je reprenais courage doucement, avec l'aide de quelqu'un je me suis achetée une voiture d'occasion, avec laquelle nous pouvions partir nous abriter, le temps d'une tempête. »

Après la virgule et le point qui sont les deux ponctèmes dominants dans tout le discours de ces femmes battues, nous avons pu constater la quasi-inexistence du point-virgule, la forte utilisation des points de suspensions et les points d'interrogations. Dans un premier temps, la quasiinexistence du « point-virgule ; " puisqu'il ne représente que $0,45 \%$ par rapport à l'ensemble de la ponctuation. Une absence due à la présence des phrases courtes et le peu de phrases composées qui nécessiteraient un recours fréquent au " point-virgule». Le second constat concerne l'utilisation des " points de suspension » à valeur de $9 \%$ et des points d'exclamation à valeur de $3 \%$, ce sont les deux ponctèmes qui apparaissent en premier sur le tableau des hautes fréquences du corpus en exprimant la subjectivité dans le récit: 1246 fréquences de « ... » au 23 ème rang et 785 fréquences de «! » au 39 ème rang. En effet, les points de suspension sont dus aux propos difficiles à exprimer, ils demandent au lecteur de deviner la suite de ce qui aurait été dit. Ils marquent la difficulté à trouver les mots pour raconter l'indicible et c'est une forme d'implicite et d'interprétation de dires. Ils représentent le silence, l'incapacité à mettre des mots sur la douleur éprouvée, la difficulté à accepter une situation anormale. Ils créent une sorte d'imagination et d'ouverture ou même, dans certaines situations ils racontent l'indicible :

« Je n'ai pas eu de rendez-vous car je n'y allais jamais au "bon moment"... [...] mais les thérapies utiles ne sont pas prises en charge par la sécurité sociale ni par les mutuelles, ou si peu...ou si cher...»

«Bravo à tous les organismes mis en place pour sauver ces femmes et ces hommes parfois.... »

Quant au point d'interrogation, nous nous demandons où se trouvent les interrogatives dans le corpus et quels sont leurs rôles ? ce sont soit des questions qui n'attendent pas de réponses, rhétoriques et ouvertes, soit des réflexions personnelles sous forme de monologues durant lesquels la femme se questionne sur une justification expliquant la stupidité de s'être laissé faire et avoir laissé passer des actes aussi graves. Soit elle se demande pourquoi cette malchance et cette injustice. Ce peut être aussi des autos-questions sur leur capacité de se projeter dans l'avenir :

« Je ne comprends pas pourquoi il me fait subir tout ca. Pourquoi dois-je subir ça? Je me pose souvent la question, ai-je fait du mal ce point-là pour être autant malheureuse ?»

« Je me demande pourquoi ai -je perdu autant de temps ???? La vie est si belle et jamais plus un homme ne posera ses mains sur moi, je crains le pire pour lui. »

«Pourquoi la justice n'a pas tenu compte des preuves incontestables que j'ai apportées et a retenu ses calomnies, mensonges, et incohérences ? Pourquoi les juges ne lui ont pas demandé des preuves de ses affirmations, se contentant de l'absence des documents ? "

\section{Conclusion}

Le témoignage a une toute autre portée que les témoignages écrits sur papier ou prononcés en dehors de la sphère numérique. Il s'agit du même objectif, toutefois un changement s'opère au niveau de la réception puisque le discours oral n'est entendu que par les auditeurs présents, et le discours écrit sur papier peut rester sans lecteur en dépit de sa possible large médiatisation par le biais d'une publication, d'un livre, d'une diffusion ou d'un enregistrement. 
Le témoignage, tout en se présentant comme un des actes persuasifs qui suscitent la croyance, y ajoute quelques conditions particulières, qui sont supposées " donner du poids » à la véracité d'une énonciation, et qui semblent en quelque sorte " objectiver » les raisons de croire. En outre, les témoignages des violences conjugales ouvrent un espace de discussion, de partage, d'immersion, permet de prendre part et de passer du rôle de lecteur à celui d'acteur actif pouvant changer le cours des choses.

Un dialogue se crée et c'est le but même de ceux qui viennent témoigner dans l'attente d'un retour positif. Les femmes battues ont usé du numérique afin de se dévoiler dans l'ordre de la reconstruction de soi, le fait de parler sur le web, de partager et de s'extérioriser sur des forums regardés et partagés diffuse une parole qui tend à modifier l'ordre du discours : une parole acceptée, lisible, lue et comprise après avoir longtemps été honteuse et taboue.

Au niveau de la forme écrite du récit, on observe de longues séquences narratives qui relatent les faits, souvent au présent qui a une valeur à la fois d'énonciation et de narration, ainsi qu'au passé composé et à l'imparfait. La quasi-absence d'utilisation du futur en dit long sur l'incapacité des victimes à se projeter sereinement dans l'avenir. Une focalisation interne nous permet de vivre le témoignage du point de vue de la personne, et de ce fait de se mettre plus facilement à sa place. Le discours rapporté, utilisé principalement pour relater les paroles de la personne violente à l'égard de la victime, nous permet d'y avoir accès et marque une certaine volonté de distanciation de la part de l'auteur du témoignage. En ce qui concerne plus précisément la ponctuation, on observe une surabondance de points de suspension, qui ne sont pas sans évoquer l'indécision, l'hésitation, l'inachevé, le non-dit, l'inaccompli, ou encore la réticence et la difficulté à mettre des mots sur le vécu. Dans le même ordre d'idées, la fréquence du pronom «ça » dénote la difficulté à dire l'indicible, la volonté de mise à distance. Le récit est dominé par la présence des pronoms personnels « je » et « il», les violences conjugales étant un drame qui se déroule généralement à huis clos entre deux personnes. De nombreux connecteurs temporels, parfois extrêmement précis - ce qui montre à quel point les fait restent gravés - émaillent le récit. En somme, le numérique est disponible sur plusieurs supports et donne naissance à des enjeux, des spécificités et des contraintes d'une nouvelle manière de mettre la souffrance en récit.

\section{Références bibliographies}

Ablali D. (2015). Sémiotique de l'épistolarité numérique d'un public en situation de souffrance in $D$. Ablali, S. Badir, D. Ducard (éd.). En tous genres. Normes, textes, médiations. Louvain-la-neuve: L'Harmattan -Academia, pp. 11-24.

Ablali D. \& Wiederspiel B. (2015). Quand le lien numérique maintient le fil narratif des personnes en souffrance, in Communication \& Langages, ${ }^{\circ} 186$, Décembre, La souffrance à proximité : écrits du mal-être d'un public en ligne, pp. 77-98.

Adam J.-M. (1987). Approche linguistique de la séquence descriptive, in Pratiques, $\mathrm{N}^{\circ} 55$.

Adam J.-M. (2011). Les textes types et prototypes, Armand Colin, (3ème edition), pp. 191-202.

Amossy R. (2014). L'éthos et ses doubles contemporains. Perspectives disciplinaires, in Langage et société, 3/2014, $\mathrm{n}^{\circ}$ 149, pp. 13-30. URL : http://www.cairn.info/revue-langage-et-societe-2014-3-page13.htm. DOI : $10.3917 / 1$ s. 149.0013 .

Anne W. (1998). L'Ere du témoin. Paris : Plon. 
Benveniste, É. (1974). Problèmes de linguistique générale II. Paris : Gallimard.

Boltanski, L. (2007). La souffrance à distance [Texte imprimé] : morale humanitaire, médias et politique ; suivi de la présence des absents. Paris : Gallimard. impr. 2007, cop. 2007.

Bonnafous, S. \& Tournier, M. (1995). Analyse du discours, lexicométrie, communication et politique in Langages, $2^{\mathrm{e}}$ année, ${ }^{\circ} 117$. Les analyses du discours en France, sous la direction de Dominique Maingueneau, pp. 67-81.

Moïse, C \& hugonnier, C. (2019). Discours homophobe » Le témoignage comme discours alternatif ». Semen - Revue de sémio-linguistique des textes et discours, Presses Universitaires de l'Université de Franche Comté (Pufc), Discours de haine dissimulée. Quelles stratégies de contre-discours?, pp.121-136. hal-02014526.

Foucault, M. (1983). L'écriture de soi, Corps écrit, 5, repris dans Dits et écrits II, 1976-1988, coll. Quarto, Paris, Gallimard, 1234-1249.

Greimas, A.-J. (1966). Pour une théorie de l'interprétation du récit mythique in Communications.

Maingueneau, D. (1996). Les termes clés de l'analyse du discours [Texte imprimé], Paris, Seuil. 1996, cop. 1996.

Maingueneau D. \& Charaudeau P. (2002). Dictionnaire d'analyse du discours, Paris, Seuil.

Maldidier, D \& Maingueneau, D. (1991), L'analyse du discours, introduction aux lectures de l'archive. In: Mots, $\mathrm{n}^{\circ} 29$. Politique et sport. Retours de Chine, sous la direction de Simone Bonnafous . pp. 108-110.

Marcoccia, M. (2004). L'analyse conversationnelle des forums de discussion : questionnements méthodologiques, Les Carnets du Cediscor [En ligne], 8 | 2004, mis en ligne le 01 novembre 2006, consulté le 05 février 2017. URL : http://cediscor.revues.org/220.

Marcoccia, M. (2012). La représentation du non verbal dans la communication écrite médiatisée par ordinateur, Communication et organisation [En ligne], 18 | 2000, mis en ligne le 27 mars 2012 ? consulté le 10 janvier 2017. URL : https://communicationorganisation.revues.org/2431.

Mayaffre, D. (2009). L'analyse $d u$ discours assistée par ordinateur disponible sur http://eprints.aidenligne-francaisuniversite.auf.org/19/1/pdf Formation Mayaffre Alexandrie dec09 .pdf.

\section{Notes de fin de document :}

\footnotetext{
${ }^{2}$ https://briserlaglace.xooit.fr/index.php

${ }^{3}$ Voir l'introduction de Mayaffre Damon au numéro 4 de : objet, méthode et contenu. Introduction, Corpus. (2005), p. 10.

${ }^{4}$ La lemmatisation désigne l'analyse lexicale du contenu d'un texte regroupant les mots d'une même famille. Chacun des mots d'un contenu se trouve ainsi réduit en une entité appelée lemme (forme canonique). Elle regroupe les différentes formes que peut revêtir un mot, soit : le nom, le pluriel, le verbe à l'infinitif, etc...

${ }^{5}$ Marcoccia Michel. «L'animation d'un espace numérique de discussion : l'exemple des forums usenet », Document numérique, vol. vol. 5, no. 3, 2001, pp. 11-26.

${ }^{6}$ Maldidier Denise, Dominique Maingueneau, L'analyse du discours, introduction aux lectures de l'archive. In: Mots, n²9, décembre 1991. Politique et sport. Retours de Chine, sous la direction de Simone Bonnafous . pp. 108-110.

${ }^{7}$ Anne Wieviorka, L'Ere du témoin, Paris, Plon, 1998. http://www.revue-silene.com/images/30/extrait_108.pdf

${ }^{8}$ Claudine Moïse, Claire Hugonnier. (2019), Discours homophobe. Le témoignage comme discours alternatif. Semen, Discours de haine dissimulée. Quelles stratégies de contre-discours? pp.121-136. hal-02014526
} 